\title{
Positive patient experience of wide awake local anaesthesia no tourniquet (WALANT) hand surgery in the government setting: a prospective descriptive study
}

\author{
Jaco J Naude, ${ }^{1 *}$ (D) Odette Koch, ${ }^{2}$ Ludwig W Schmidt, ${ }^{1}$ Theo LB le Roux ${ }^{2}$ \\ ${ }^{1}$ Steve Biko Academic Hospital, Department of Orthopaedic Surgery, University of Pretoria, South Africa \\ 21 Military Hospital, Department of Orthopaedic Surgery, University of Pretoria, South Africa; Netcare Pretoria East Hospital \\ ${ }^{*}$ Corresponding author: jacojnaude@yahoo.com
}

Citation: Naude JJ, Koch O Schmidt LW, Le Roux TLB. Positive patient experience of wide awake local anaesthesia no tourniquet (WALANT) hand surgery in the government setting: a prospective descriptive study. SA Orthop J 2021;20(3):141-146. http://dx.doi.org/10.17159/23098309/2021/v20n3a1

Editor: Dr Duncan McGuire, University of Cape Town, Cape Town, South Africa

Received: June 2020

Accepted: November 2020

Published: August 2021

Copyright: @ 2021 Naude JJ.

This is an open-access article distributed under the terms of the Creative Commons Attribution Licence, which permits unrestricted use, distribution and reproduction in any medium, provided the original author and source are credited.

Funding: No funding was received for this study.

Conflict of interest: The authors declare they have no conflicts of interest that are directly or indirectly related to the research.

\section{Abstract \\ Background}

The purpose of this study was to establish a subjective patient experience with wide awake local anaesthesia no tourniquet (WALANT) procedures performed in the institution from May 2019 to March 2020. WALANT surgery was initiated to improve standard operating procedure and to decrease theatre burden.

\section{Methods}

This prospective, descriptive study included 100 patients with a mean age of 59 years who required either a carpal tunnel or trigger finger release. The patients' pain experience was documented on the visual analogue scale (VAS) for the local anaesthetic injection and the surgical procedure. Overall experience was assessed on the patient's preference to have the procedure performed by the WALANT method or the conventional method.

\section{Results}

One hundred patients were included, of which 67 had medical comorbidities. The mean VAS score was $1.5(\mathrm{SD} \pm 1.6)$ with pain on injection. The mean VAS pain score during the surgical procedure was $0.2(S D \pm 0.7)$. One hundred per cent of patients $(100 / 100)$ felt they would do the WALANT outpatient procedure again instead of admission to hospital and surgery in the theatre. Two complications occurred related to wound care problems, and were successfully managed. None of the patients required reoperations for incomplete release of the carpal tunnel or trigger finger surgery.

\section{Conclusion}

The results of this study suggest that minor hand surgery using the WALANT protocol can be performed effectively and with high patient satisfaction rates in the orthopaedic outpatient clinic, and is a useful tool in the skillset of a hand surgeon.

Level of evidence: Level 4

Keywords: WALANT, hand surgery, trigger finger, carpal tunnel release

\section{Introduction}

Carpal tunnel surgery and trigger finger release are commonly performed procedures in hand surgery, with over 400000 procedures per year in the United States. ${ }^{1}$ However, with increasing cost and the limited resources faced by many government institutions, some surgeons have looked at alternative, less expensive and more accessible venues to perform minor procedures. ${ }^{2}$

There has been an increased interest in and experience with performing surgical procedures on the hand and wrist utilising local anaesthesia in an unsedated patient. These surgical procedures can be safely performed on an outpatient basis using wide awake local anaesthetic no tourniquet (WALANT). ${ }^{3}$ This technique has been popularised and well described by plastic surgery. ${ }^{3}$
WALANT surgery makes it possible to do smaller cases of the hand in a more cost-effective manner in a clinic or outpatient setting, as shown in studies in America and Canada. ${ }^{1,2}$ Patient satisfaction is high with this technique because the pre-surgical survey is not needed, and post-anaesthetic recovery is rapid, due to a reduction in anaesthetic-related complications. ${ }^{2,4-8}$ Even though the success of WALANT surgery is well recorded in developed world countries, limited data exists from South Africa, and even more so in the government setting. The authors postulated that this method of surgery could solve problems currently existing in many state hospitals, including a large number of patients with limited theatre availability, multiple anaesthetic cancellations, long waiting lists and limited priority of cold/elective surgery. 
The benefits of the WALANT approach include:

- No sedation and no tourniquet increase patient comfort and convenience

- Simpler draping decreases surgical time; patients can have hand surgery in much the same way as a minor procedure at the dentist

- Eliminating the anaesthetic component decreases treatment time for minor procedures such as carpal tunnel releases, improving efficiency and workflow

- During a surgical procedure, the patient can flex and extend fingers to show complete release, assisting in postoperative compliance

- Surgeons can discuss postoperative care and rehabilitation with the patient, improving patient compliance

- Immediate patient discharge postoperatively decreases the recovery process ${ }^{3,4}$

The current main theatre protocol at the institution includes admission at 7 o'clock the morning before surgery. Preoperative workup for theatre for all patients above 40 years of age, regardless of the procedure, includes the following: chest X-ray, electrocardiogram (ECG), lung function tests and blood workup, which consists of a minimum of FBC and U\&E. If indicated, extended biochemistry, including thyroid functions, calcium, magnesium and phosphate levels and liver function tests are conducted. An anaesthetic registrar or medical officer sees the patient the afternoon before surgery, and possibly asks for more tests, often including heart sonars and even angiograms. If further investigations are not completed before the planned procedure, the patient is cancelled and rescheduled after complete workup. Once the required test results are available, the patient will be null per os from 10 o'clock the evening before surgery and be operated the following day, after which they will be discharged the same day postoperatively.

Hand surgeons and anaesthetists in government are currently reluctant to start using WALANT. In the protocol without WALANT, there is unnecessary preoperative workup for minor hand procedures, which waste valuable resources, workforce and money. Incorporating the principle of 'first do no harm', the authors had to prove to the management of the hospital that the patients would not be caused damage and suffering as a result of WALANT.

Therefore, the objective of the current study was to establish a subjective patient experience with WALANT procedures performed at the institution from May 2019 to March 2020. The secondary objective was to assess if there were any differences in the level of pain during carpal tunnel and trigger finger release, or between males and females.

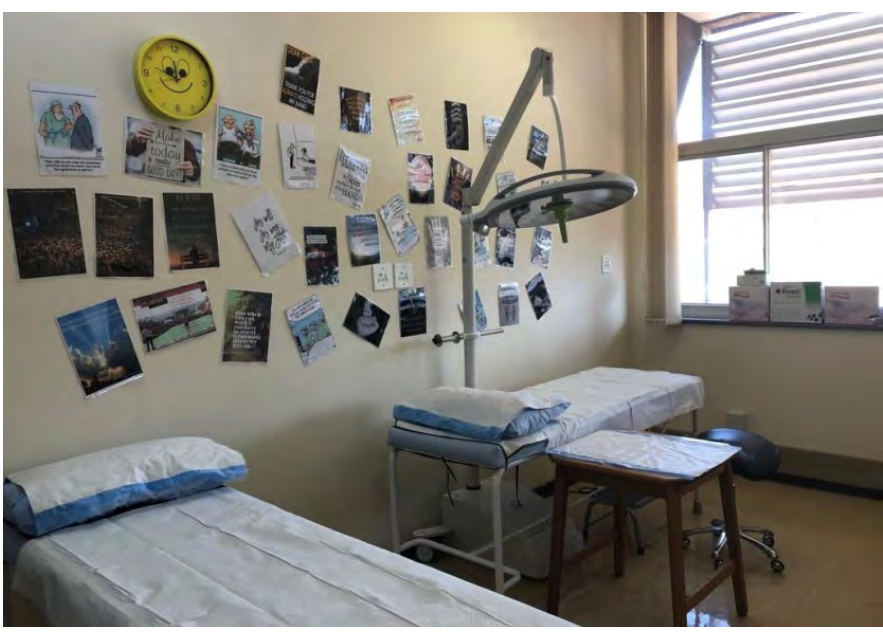

Figure 1. Image of WALANT theatre room in the orthopaedics outpatient department

\section{Patients and methods}

\section{Study design and research methodology}

This prospective, descriptive study was done over ten months, from May 2019 to March 2020, and included 100 patients with trigger fingers or carpal tunnel syndrome requiring surgical release. Consecutive inclusion was applied as the sampling method.

\section{Patient description and inclusion criteria}

Patients were included in the study if they required surgery for carpal tunnel release or trigger finger release; were above the age of 18 years; with or without any associated medical comorbidities. Patients were excluded from the study if they did not give consent; were anxious about the local procedure; were not suitable for WALANT surgery based on the surgeon's discretion. Patient demographics, including age, sex, diagnosis and WALANT procedure, were recorded.

The research and ethics committee of the institution approved the study before study commencement. Patients were enrolled voluntarily. All patients signed and dated the patient information leaflet and informed consent document on the day of the surgery.

\section{Procedure description}

A room in the orthopaedic outpatient department was equipped for the surgical procedures (Figure 1), with a specialised hand surgeon and two registrars performing all of the surgeries. Cases were limited to eight per day as the hospital has a total of 12 handsets. The main theatre had access to the other four handsets. These cases were performed in the clinic between 8 and 12 o'clock in the morning. Central sterile services department (CSSD) supplied the sterile sets on the morning of the surgery. Patients reported at the clinic at 8 o'clock on the morning of the procedure, and consent for the surgery was signed. The patients were infiltrated with local anaesthetics. The infiltrations were performed sequentially, infiltrating three cases from 8 o'clock, and thereafter infiltrating the next cases between surgeries as the list progressed. All injections were performed with the patients on a bed in the supine position as some patients may experience a vasovagal attack with lightheadedness from the infiltration.

The volume of $20 \mathrm{ml}(20 \mathrm{cc})$ infiltration fluid required for carpal tunnel release under WALANT surgery (Figures 2 and 3) consists of: ${ }^{9}$

- Lignocaine 1\% (10 ml)

- Adrenalin 1:100 $000(2 \mathrm{ml})$

- Bicarbonate $8.4 \%: 1 \mathrm{ml}$ in each $10 \mathrm{ml}$ infiltration to increase the $\mathrm{pH}$ from 4.7 to 7.4 . This decreases the burning pain during infiltration (2 $\mathrm{ml})$

- Sterile water $(6 \mathrm{ml})$

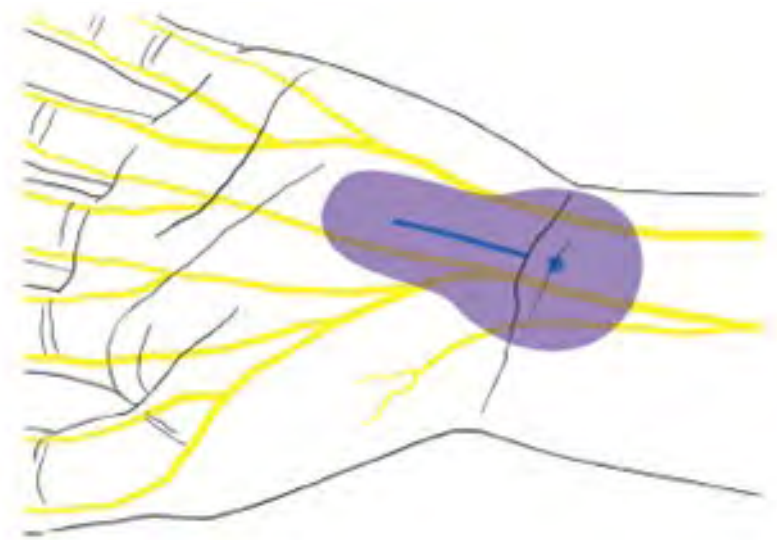

Figure 2. Carpal tunnel infiltration location ${ }^{9}$ (used with permission from Lalonde and Wong) 
A total of $4 \mathrm{ml}$ of the same mixture of infiltration fluid was used for every trigger finger release (Figure 4). A 27- or 30-gauge needle was used to ensure minimal pain during insertion. The small gauge of the needle slowed the injection down, making it less painful. Sensory noise was created by proximally pinching the injection site. The needle was inserted perpendicular to the skin, not injecting intradermally to minimise pain. The "blow before you go' injection technique was followed. The solution was injected slowly before the needle progressed deeper into the tissue. Using this technique, the nerve endings were blocked before the needle reached the deeper tissue. The following injections stayed within $10 \mathrm{~mm}$ of indurated areas to decrease pain.

All injections were below the safe limit of $7 \mathrm{mg} / \mathrm{kg}$ for lignocaine injection. The patients were then given a resting period of $30 \mathrm{~min}-$ utes to give the epinephrine and anaesthesia time to take effect.

Patients were operated in a sterile field, prepping the hand with chlorhexidine and alcohol. Three towels or drapes were used per procedure (two to drape, and one rolled-up towel as positioner under the hand), as well as sterile gloves and masks. The surgeons were not gowned or capped. During the procedure, the surgeon communicated with the patient, relying on verbal feedback. No vital signs were monitored during the process. The resuscitation trolley was within reach and regularly checked. Sterile dressings were placed, comprising gauze without the use of elastic bandages. No

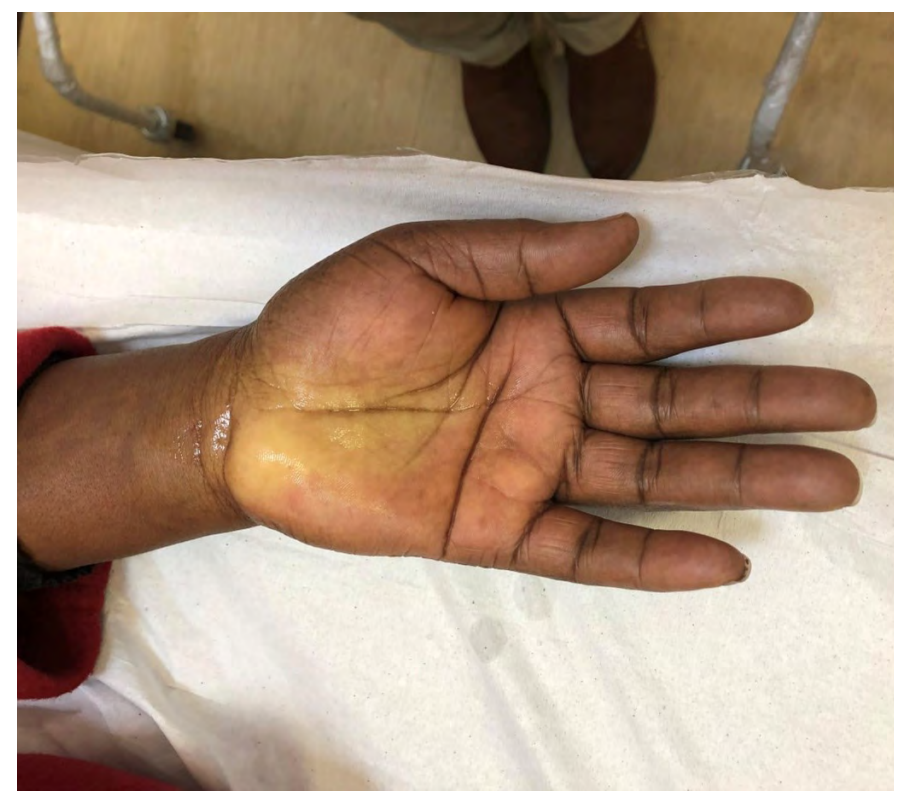

Figure 3. Blanching after carpal tunnel infiltration

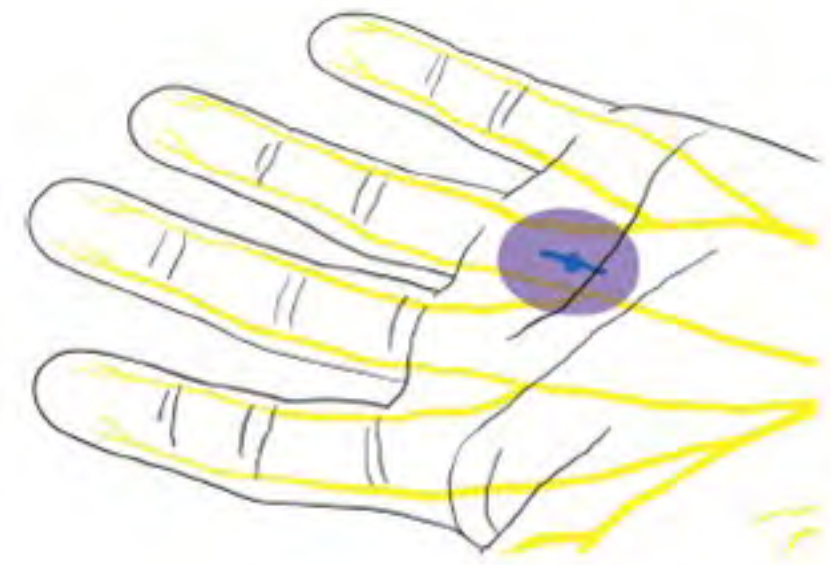

Figure 4. Trigger finger infiltration location ${ }^{9}$ (used with permission from Lalonde and Wong) prophylactic antibiotics were given. In the case of trigger finger release, the patient was asked to flex and extend the finger to ensure complete release on the table. The occupational therapist gave the patient information regarding a home programme for hand therapy during the procedure. Dressings were removed at home, and a follow-up consultation was scheduled two weeks following the procedure for all cases.

\section{Outcome measures}

Patients were asked to grade the pain on the VAS (numerical and Wong-Baker pain score) in order to establish the patients' subjective experience at completion of the surgery. Two separate questions were used to grade the pain according to VAS:

1. Grade the pain of the injection during infiltration on a scale of $0-10$.

2. Grade the pain during the surgical procedure on a scale of $0-10$.

Additionally, an open-ended question was asked to give context to the WALANT surgery experience. Patients were asked if they had to have a similar procedure done in the future, if they would do it via the WALANT protocol again, or if they would rather do it via the main theatre protocol, with early admission, full workup and surgery under general anaesthesia.

\section{Statistical analysis}

One hundred patients were included with trigger fingers and carpal tunnel syndromes that required surgical release from May 2019 to March 2020. Means, standard deviations, medians and interquartile ranges were used to describe continuous variables. Frequencies and proportions were used to describe categorical variables, including the percentage of patients who would have the procedure again. The Mann-Whitney non-parametric test was used to compare pain ratings between groups since the pain ratings were not normally distributed and unbalanced. Statistical significance was taken at $5 \%(p=0.05)$. Tests were conducted using STATA 15.

\section{Results}

One hundred patients underwent carpal tunnel and trigger finger release under WALANT at the institution from May 2019 until March 2020. All patients were followed up until wounds healed, complications resolved or the clinical condition improved, with most patients discharged from the clinic at two weeks. No patient was lost to follow-up as these patients can only be seen in the institution or at the institution's sickbays due to the nature of the specific medical aid. No revision surgery to date was required for incomplete release of carpal tunnel syndrome or trigger finger. No patients were excluded from the study.

The study included 57 females and 43 males, with a mean age of 59 years, ranging from 28 to 82 years $(S D \pm 12.8)$. Sixty-seven patients had comorbidities, mostly consisting of hypertension, diabetes and hypercholesterolaemia (Table I).

The mean VAS score with pain on injection was 1.5, ranging from 0 to $9(S D \pm 1.6)$. The mean VAS pain score during the surgical procedure was 0.2 , ranging from 0 to $5(\mathrm{SD} \pm 0.7)$ (Table II). There were no significant differences in pain between the diagnosis of carpal tunnel syndrome and trigger finger release with pain on injection $(p=0.45)$ or pain during the procedure $(p=0.69)$. There was no significant difference between males and females with pain on injection $(p=0.32)$ or pain during the procedure $(p=0.74)$.

Asked if they would prefer to do the surgery again under WALANT or rather in theatre under general anaesthetics, $100 \%$ of patients reported that they would prefer to have it under WALANT.

There were no cases of artery or nerve penetration, no terminal finger cyanosis or ischaemic skin changes. No local intraoperative 
Table I: Various patient comorbidities in the study population

\begin{tabular}{lc} 
Comorbidity & Total \\
\hline Diabetes & 14 \\
\hline Hypertension & 44 \\
\hline Hypercholesterolaema & 17 \\
\hline Gout & 3 \\
\hline Asthma & 1 \\
\hline COPD/emphysema & 3 \\
\hline GORD & 1 \\
\hline Porphyria & 1 \\
\hline Psoriasis & 1 \\
\hline Tracheal stenosis & 1 \\
\hline Peripheral vascular disease & 1 \\
\hline Cardiac pathology (including MI, valve pathology, & 7 \\
\hline cardiac bypass, pacemakers) & 1 \\
\hline Previous scoline apnoea & 1 \\
\hline Multiple allergies & 3 \\
\hline Rheumatoid arthritis & 1 \\
\hline
\end{tabular}

complications occurred. There was one incident of a patient who had a vasovagal response after the infiltration, which resolved with standard treatment principles. No patients needed to be cancelled or changed to other anaesthetic methods due to failure of WALANT.

One patient developed chronic regional pain syndrome (CRPS) of the hand after a carpal tunnel release for which they received occupational therapy. The symptoms of the CPRS and the carpal tunnel resolved.

Two adverse events related to wound complications occurred that were not infiltration related. Both of the cases required admission to hospital and secondary surgery. The first case was a 71-year-old male, who was on double platelet therapy (Pradaxa and Cardiodisprin), with two previous cardiac bypasses and a cardiac ejection fraction of $35 \%$. He had a carpal tunnel and trigger finger release under WALANT. Five days postop, the patient was admitted to the hospital for a haematoma in the forearm. The patient required secondary surgery to debride the forearm. The clinical symptoms of the presenting complaint and wound complication resolved. The second case was a 60 -year-old female who attended the clinic two weeks after carpal tunnel release. The patient's wound was assessed as clean, and sutures were removed in the procedure room by nursing staff. The nursing staff removed the most proximal mattress suture with difficulty. The patient was admitted three days later (17 days after the surgery) with a wound infection in the forearm. She required a secondary procedure for debridement of the infection in the forearm. She received intravenous antibiotics and was admitted for seven days. The wound infection resolved.

\section{Discussion}

The current study confirmed that positive subjective patient experiences were universally reported when WALANT surgery was used for minor hand procedures. One hundred per cent of patients were prepared to undergo second surgeries using this method. Acceptable VAS pain scores were also noted. WALANT surgery is widely accepted for many wrist and hand surgeries. ${ }^{7,10-12}$ Rhee et al. ${ }^{6}$ had $73 \%$ of patients estimate their pain scores as less painful than a dental procedure. They had a mean maximum VAS pain score for carpal tunnel release of 5.0/10 $( \pm 3.1)$. This was also their first experience with WALANT surgery. The researchers postulate that the meticulous technique used during injection ensured low pain VAS scores, with a mean of 1.5 during injection, as well as during surgery, with a mean of 0.2 . Other techniques to decrease pain on injection include warming the local anaesthetic, distracting the patient or the area of injection, stabilising the syringe, thus preventing the needle from wobbling, and pausing after $0.5 \mathrm{ml}$ injection to let it take effect, then injecting an additional $2 \mathrm{ml}$ before moving the needle. It is crucial to learn from each infiltration via patient feedback. ${ }^{5}$ The effectiveness of vasoconstriction of the adrenaline lasts four to five hours. ${ }^{4}$

Older patients with extensive comorbidities and chronic illnesses may have physiological derangements and decompensation precipitated by anaesthesia. In this study, there were 67 patients with medical comorbidities, potentially complicating general anaesthesia. The current study included three patients who were not fit for general anaesthetics due to their medical comorbidities.

By allowing patients to come to the outpatient department the morning of surgery, and be discharged immediately after surgery, the researchers have significantly decreased the length of stay compared to the standard protocol of staying over in hospital for one night. ${ }^{1-3,6,13}$ All of the above advantages assisted with patients' satisfaction, ensuring that all of the patients opted to have the WALANT procedure again if need be. As demonstrated in the current study, and as advocated by the WALANT University, patients are part of the surgery. ${ }^{14}$ They are able to see that their finger has a full range of motion and no longer locks. This sense of inclusion on the part of the patient might add further to the positive patient experience.

It was once widely believed that injected epinephrine frequently caused finger ischaemia and necrosis. This goes back to the

Table II: Results on VAS pain scores comparing diagnosis and age

\begin{tabular}{|c|c|c|c|c|c|c|c|c|}
\hline Diagnosis & Variable & Min & $\operatorname{Max}$ & SD & Median & P25 & P75 & IQR \\
\hline \multirow{3}{*}{$\begin{array}{l}\text { Carpal tunnel syndrome } \\
(\mathrm{n}=69)\end{array}$} & Age (years) & 28 & 82 & 13.9 & 58 & 48 & 69 & 21 \\
\hline & Injection VAS & 0 & 9 & 1.7 & 1 & 0 & 0 & 2 \\
\hline & Procedure VAS & 0 & 2 & 0.4 & 0 & 0 & 0 & 0 \\
\hline \multirow{3}{*}{$\begin{array}{l}\text { Trigger finger \& carpal tunnel } \\
\text { syndrome }(n=6)\end{array}$} & Age (years) & 46 & 77 & 11.6 & 70 & 61 & 76 & 15 \\
\hline & Injection VAS & 0 & 5 & 1.9 & 1.5 & 0 & 2 & 2 \\
\hline & Procedure VAS & 0 & 3 & 1.2 & 0 & 0 & 1 & 1 \\
\hline \multirow[t]{3}{*}{ Trigger finger $(n=25)$} & Age (years) & 44 & 78 & 9.4 & 59 & 51 & 65 & 14 \\
\hline & Injection VAS & 0 & 4 & 1.4 & 1 & 0 & 2 & 2 \\
\hline & Procedure VAS & 0 & 5 & 1.0 & 0 & 0 & 0 & 0 \\
\hline \multirow[t]{3}{*}{ Total $(n=100)$} & Age (years) & 28 & 82 & 12.8 & 59 & 49 & 69 & 20 \\
\hline & Injection VAS & 0 & 9 & 1.6 & 1 & 0 & 2 & 2 \\
\hline & Procedure VAS & 0 & 5 & 0.7 & 0 & 0 & 0 & 0 \\
\hline
\end{tabular}


1950s when procaine was used, which caused isolated cases of finger ischaemia and necrosis. ${ }^{15}$ Procaine was replaced by lignocaine, which was introduced in 1948. Procaine has a pH of 3.4 and can decrease to a $\mathrm{pH}$ of 1 if stored for long periods. This acidity can cause finger necrosis and is not associated with the use of epinephrine. ${ }^{16}$ Large studies have confirmed that clinical epinephrine can be used without inducing infarction. ${ }^{1,15,17-21}$ This has also been shown with high-dose (1:1000) epinephrine injections in the fingers. ${ }^{19}$ In WALANT procedures, 1:100 000 epinephrine is used. Level 1 evidence has shown that phentolamine, an alphablocker, reliably reverses epinephrine vasoconstriction in the finger. ${ }^{22}$ However, its use is seldom required in clinical practice. In this study, no patient had signs of finger ischaemia, and no patient required phentolamine to reverse the vasoconstrictor effect.

One of the questions around moving surgical procedures out of theatre is the incidence of infection and sterility fields. LeBlanc et al. ${ }^{23}$ looked at 1504 consecutive patients treated with open carpal tunnel release in a sterile field, outside of the theatre setting. Six superficial infections were reported; four of those six patients received oral antibiotics, and two were not severe enough to require antibiotics. Not one deep postoperative wound infection was encountered, and no patient received or required an incision and drainage or intravenous antibiotics. Their study, therefore, had a superficial infection rate of $0.4 \%$ and a deep infection rate of $0 \%$ following a minor surgical procedure outside of the theatre. Rhee et al. ${ }^{6}$ had a superficial infection rate of $3 \%$. In the current study, there were two patients $(2 \%)$ who developed wound complications requiring readmission and follow-up surgery, one of which was a deep infection of the forearm. The similar infection rate to Rhee's study may indicate that there is a learning curve in performing successful WALANT surgery repeatedly. The implementation of a new technique requires a successful, reproducible system to allow procedures to run smoothly and to minimise complications. This also indicates the importance of monitoring oneself during the injection procedure to improve accuracy and decrease patient discomfort. Infection risk remains minimal and comparable to the in-theatre setting. ${ }^{23}$

It has been shown that outpatient procedures like carpal tunnel releases have an improved efficiency compared to in-theatre procedures. Up to 20 cases can be done in an outpatient setting in a ten-hour day, compared to only ten cases in a theatre setting, as shown in a study in the United States. ${ }^{1}$ In Canada, a similar study has shown improved efficacy by doing nine cases of carpal tunnel release in an outpatient setting in a three-hour block, compared to four cases in theatre. ${ }^{2}$ Both these studies have also shown outpatient surgery cost savings and efficiency improvement in their respective settings. The researchers managed to perform eight cases on a morning list in the outpatient clinic between 8 and 12 o' clock. This is limited to eight to prevent system overload and because we only have eight handsets available from CSSD. The researchers have noticed that this has caused the elective theatre waiting time to decrease. By removing the smaller procedures from the elective list, the average waiting time for elective surgery has been reduced from eight to 12 weeks, to on average four to six weeks. The WALANT procedures also decrease the burden on an already over-stressed healthcare system, not only with regard to costs but also personnel usage, as only one staff nurse is required for the list.

LeBlanc also considered the possible environmental impact of using the main theatre sterility. ${ }^{14}$ Although they have not studied it in detail, they estimated that the amount of garbage that is generated by the main operating theatre sterility is at least ten times that of minor procedure field sterility. ${ }^{14}$ The current study provided similar findings, as the total refuse after a morning list in the outpatient department has decreased to one bag. This decrease in refuse will have a positive impact on the environment in the long run.
The limitations of the study were the relatively small number of patients compared to similar studies. The study is, however, sufficient to indicate the positive patient experience with WALANT surgery due to the definitive patient response. The sample population was selected from only one hospital. There may still be differences in the larger population and in other government hospitals, leading to some selection bias. This study only reports on the subjective experience of a small group of patients, and socioeconomic, educational and cultural factors were not considered. The higher rate of complications in this study can be explained by the learning curve of the WALANT protocol. It is evident that the current theatre protocol at the institution is excessive for minor procedures of the hand. There is room for improvement, specifically in the government setting.

\section{Conclusion}

This study confirmed that the patients at the institution had a positive subjective experience when WALANT surgery was used for minor hand surgery. One hundred per cent of patients were prepared to undergo second surgeries using this method. Acceptable VAS pain scores were also noted. This study shows that there are significant benefits to WALANT surgery and that WALANT surgery is a useful tool in the skillset of a hand surgeon.

\section{Ethics statement}

The authors declare that this submission is in accordance with the principles laid down by the Responsible Research Publication Position Statements as developed at the 2nd World Conference on Research Integrity in Singapore, 2010.

All procedures performed in studies involving human participants were following the ethical standards of the institutional and/or national research committee and with the 1964 Helsinki declaration and its later amendments or comparable ethical standards. Ethics approval was gained from the 1MHREC (1 Military Hospital Research and Ethics Committee) $1 \mathrm{MH} / 302 / 6 / 02.05 .2019$. Informed consent was obtained from all individual participants included in the study.

\section{Declaration}

The authors declare authorship of this article and that they have followed sound scientific research practice. This research is original and does not transgress plagiarism policies.

\section{Author contributions}

JJN: Study conceptualisation and design, data collection, data analysis, manuscript preparation

OK: Study conceptualisation and design, data collection, data analysis, manuscript preparation

LWS: Data collection and data analysis

TLBR: Study conceptualisation and design and liaising between the 1MHREC and the investigators

\section{ORCID}

JJ Naude iD https://orcid.org/0000-0003-1448-4272

O Koch (iD https://orcid.org/0000-0003-1871-9569

LW Schmidt (iD https://orcid.org/0000-0002-1144-6433

TLB le Roux (iD https://orcid.org/0000-0003-1871-9569

\section{References}

1. Chatterjee A, McCarthy JE, Montagne SA, et al. A cost, profit and efficiency analysis of performing carpal tunnel surgery in the operating room versus the clinic setting in the United States. Ann Plast Surg. 2011;66(3):245-48. https:// doi.org/10.1097/SAP.0b013e3181db7784

2. LeBlanc MR, Lalonde J, Lalonde DH. A detailed cost and efficiency analysis of performing carpal tunnel surgery in the main operating room versus the ambulatory setting in Canada. Hand. 2007;2(4):173-78. https://doi.org/10.1007/ s11552-007-9043-5.

3. Lalonde D, Eaton C, Armadio PC, Jupiter JB. Wide awake hand and wrist surgery: A new horizon in outpatient surgery. AAOS instructional course lectures, Vol 64. 2015

4. Tang JB, Gong KT, Zhu L, et al. Performing hand surgery under local anesthesia without a tourniquet in China. Hand Clin. 2017;33:415-24. https:// doi.org/10.1016/j.hcl.2017.04.013. 
5. Strazar RA, Leynes GP, Lalonde DH. Minimising the pain of local anesthesia injection. Plastic Reconstr Surg. 2013;132:675-84. https://doi.org/10.1097/ PRS.0b013e31829ad1e2.

6. Rhee PC, Fischer MM, Rhee LS, et al. Cost savings and patient experiences of a clinic-based, wide-awake hand surgery program at a military medical centre: a critical analysis of the first 100 procedures. J Hand Surg Am. 2017:42:e13947. https://doi.org/10.1016/j.jhsa.2016.11.019.

7. Lalonde $\mathrm{DH}$. 'Hole-in-one' local anesthesia for wide-awake carpal tunnel surgery. Plast Reconstr Surg. 2010;126:1642-44. https://doi.org/10.1097/ PRS.0b013e3181f1c0ef.

8. Steiner MM, Calandruccio JH. Use of wide-awake local anesthesia no tourniquet in hand and wrist surgery. Orthop Clin N A.m 2018;49:63-68. https:// doi.org/10.1016/j.ocl.2017.08.008.

9. Lalonde $\mathrm{DH}$, Wong $\mathrm{A}$. Dosage of local anesthesia in wide awake hand surgery J Hand Surg. 2013;38A:2025-28. https://doi.org/10.1016/j.jhsa.2013.07.017.

10. Lalonde $D$, Higgins $A$. Wide awake flexor tendon repair in the finger. Plast Reconstr Surg Glob Open. 2016;4(7):e797. https://doi.org/10.1097/ GOX.0000000000000756.

11. Lalonde $\mathrm{DH}$. Wide-awake extensor indicis proprius to extensor pollicis longus tendon transfer. J Hand Surg Am. 2014;39:2297-99. https://doi.org/10.1016/j. jhsa.2014.08.024

12. Gregory S, Lalonde DH, Fung LL. Minimally invasive finger fracture management: wide-awake closed reduction, K-wire fixation, and early protected movement. Hand Clin. 2014;30:7-15. https://doi.org/10.1016/j. hcl.2013.08.014.

13. Flatt AE. Tourniquet time in hand surgery. Arch Surg. 1972;104:190-92. https:// doi.org/10.1001/archsurg.1972.04180020070013.

14. WALANT University. WALANT Surgery. [Online] Available from: https://walant. surgery/walant-university/. Accessed 29 Apr 2020.

15. Lalonde D, Martin A. Epinephrine in local anesthesia in finger and hand surgery: the case for wide-awake anesthesia. J Am Acad Orthop Surg. 2013;21:443-47. https://doi.org/10.5435/JAAOS-21-08-443.

16. Pires Neto PJ, Moreira LA, Las Casas PP. Is it safe to use local anesthesia with adrenaline in hand surgery? WALANT technique. Rev Bras Orhop. 2017:52:383-89. https://doi.org/10.1016/j.rboe.2017.05.006

17. Lalonde $\mathrm{DH}$, Bell $\mathrm{M}$, Benoit $\mathrm{P}$, et al. A multicentre prospective study of 3110 consecutive cases of elective epinephrine use in the fingers and hand: The Dalhousie Project clinical phase. J Hand Surg Am. 2005;30(5):1061-37. https:// doi.org/10.1016/j.jhsa.2005.05.006.

18. Chowdhry S, Seidenstricker L, Cooney DSS, et al. Do not use epinephrine in digital blocks: Myth or truth? Part II. A retrospective review of 1111 cases. Plast Reconstr Surg. 2010;126(6):2031-34. https://doi.org/10.1097/ PRS.0b013e3181f44486.

19. Fitzcharles-Bowe $\mathrm{C}$, Denkler $\mathrm{KA}$, Lalonde $\mathrm{DH}$. Finger injection with high dose (1:1000) epinephrine: does it cause finger neurosis and should it be treated? Hand.2007;2(1):5-11. https://doi.org/10.1007/s11552-006-9012-4.

20. Muck AE, Bebarta VS, Borys DJ, Morgan DL. Six years of epinephrine digital injections: Absence of significant local or systemic effects. Ann Emerg Med. 2010;56(3):270-74. https://doi.org/10.1016/j.annemergmed.2010.02.019.

21. Tang JB, Zhou X, Pan ZJ, et al. Strong digital flexor tendon repair, extension flexion test, and early active flexion: Experience in 300 tendons. Hand Clin. 2017;33:455-63. https://doi.org/10.1016/j.hcl.2017.04.012.

22. Nodwell T, Lalonde $\mathrm{DH}$. How long does it take phentolamine to reverse adrenaline induced vasoconstriction in the finger and hand: A prospective, randomised, blinded study: The Dalhousie project experimental phase. Can $J$ Plast Surg. 2003;11(4):187-90.

23. LeBlanc MR, Lalonde $\mathrm{DH}$, Thoma $\mathrm{A}$ et al. Is main operating room sterility really necessary in carpal tunnel surgery: A multicentre prospective study of minor procedure room field sterility surgery. Hand. 2011;6(1):60-63. https://doi. org/10.1007/s11552-010-9301-9. 